\title{
Role of quinine-sensitive ion channels in volume regulation in boar and bull spermatozoa
}

\author{
A. M. Petrunkina ${ }^{1}$, R. A. P. Harrison ${ }^{2}$, M. Hebel ${ }^{1}$, K. F. Weitze ${ }^{1}$ \\ and $\mathrm{E}$. Töpfer-Petersen ${ }^{1}$ \\ ${ }^{1}$ Institute for Reproductive Medicine, School of Veterinary Medicine Hannover, Bünteweg \\ 15, 30559 Hannover, Germany; and ${ }^{2}$ Laboratory of Gamete Signalling, \\ The Babraham Institute, Babraham, Cambridge CB2 4AT, UK
}

The ability to reverse swelling caused by hypo-osmotic stress is an important cell function; in spermatozoa, it is likely to be of consequence during ejaculation and also during the thawing process that terminates cryopreservation. In this study, the time course of boar and bull sperm volume changes after exposure to hypoosmotic conditions at $39^{\circ} \mathrm{C}$ was recorded. Cell volume measurements of washed sperm suspensions were performed electronically in Hepes-buffered saline solutions of 300 and 180 mosmol kg-1 containing $2.5 \mathrm{mmol}$ $\mathrm{K}^{+} \mathrm{I}^{-1}$. Treatment with quinine in the presence or absence of the potassium ionophore valinomycin was used to determine whether potassium channels were involved in the reversal of swelling. After exposure to hypo-osmotic conditions, both bull and boar spermatozoa showed initial swelling (up to $200 \%$ and $140 \%$ of initial volume, respectively, within $5 \mathrm{~min}$ ), which was subsequently partially reversed (to about $150 \%$ and $120 \%$, respectively, after $20 \mathrm{~min}$ ). Incubation with quinine led to an increase in swelling in both species. However, bull sperm volume was already maximal (up to $294 \%$ ) after $30 \mathrm{~s}$ and declined thereafter, whereas boar sperm volume increased slowly to a maximum of about $220 \%$ after $20 \mathrm{~min}$. Valinomycin treatment caused quinine-induced swelling in bull spermatozoa to decrease rapidly to control (no quinine, no valinomycin) values, whereas in quinine-treated boar spermatozoa it had an opposite, enhancing effect. Interpreting these results in the light of data from studies by others on a variety of cell types, it is proposed that swelling-activated potassium channels are involved in regulatory volume decrease in both species of spermatozoa, but that boar spermatozoa may contain fewer swelling-activated chloride channels than do bull spermatozoa.

\section{Introduction}

There has long been interest in the response of spermatozoa to aniso-osmotic conditions. Initially it was thought that spermatozoa, unlike other cell types, did not swell in a hypoosmotic environment. However, Drevius and Eriksson (1966) and Drevius (1972) demonstrated that these cells do swell and that over a relatively wide range of tonicities they behave as perfect osmometers (swelling is proportional to the degree of hypotonicity). During its life as a free cell, a spermatozoon experiences considerable changes in its environment, most notably during maturation within the epididymis and at ejaculation (Cooper, 1986). As a spermatozoon passes down the epididymis, it encounters a major decrease in $\left[\mathrm{Na}^{+}\right]$and a major increase in $\left[\mathrm{K}^{+}\right]$, and a replacement of $\mathrm{Cl}^{-}$with organic osmolytes. The osmotic pressure of the luminal fluid varies down the length of the duct but remains significantly hyperosmotic in the distal cauda. Therefore, on deposition in the female tract, a spermatozoon will experience hypoosmotic stress as it encounters an environment of osmolality similar to that of blood plasma.

Email: Anna.Petrounkina@tiho-hannover.de
Significant changes in cell volume tend to have profound effects on cell function, through relative dilution or concentration of the intracellular components, as well as local distortion of structural elements (for example, cytoskeleton and plasma membrane) (for review see Lang et al., 1998). For example, it has been shown that in spermatozoa flagellar motility is lost irreversibly beyond a certain limited osmotic stress (see Gilmore et al., 1998). Given the potentially serious consequences of excessive volume changes in response to varying osmotic environments, it is not surprising that studies on many animal cell types have revealed a widespread ability to maintain cell volume in the face of osmotic stress (Hoffmann and Dunham, 1995). Therefore, spermatozoa would be expected to possess volume regulatory abilities, in particular the ability to moderate or nullify swelling caused by hypo-osmotic shock (regulatory volume decrease).

Cell volume is essentially determined by the intracellular content of osmotically active solute relative to the osmolarity of the extracellular fluid. Thus, volume regulation under aniso-osmotic conditions essentially involves the activation of processes for rapid net transfer of osmolytes into or out of the cell, so as to re-establish osmotic equilibrium (see 
reviews by Grinstein et al., 1984; Sarkadi and Parker, 1991; Al-Habori, 1994; O’Neill, 1999). Relatively little is known about the details of the regulatory mechanisms in spermatozoa. Kulkarni et al. (1997) reported the presence of channels in bull spermatozoa through which $\mathrm{K}^{+}$was released in response to hypo-osmotic shock; by blocking these channels with quinine, it could be shown that they played a major and rapid role in moderating the swelling response. More recently, while studying mouse spermatozoa exposed to hypo-osmotic conditions, Yeung et al. (1999) induced swelling-associated changes by treatment with inhibitors of anion channels as well as $\mathrm{K}^{+}$channels.

That regulatory volume decrease is indeed an important physiological function was demonstrated by parallel studies of spermatozoa from c-ros tyrosine kinase receptor knockout mice. Yeung et al. (1999) found that these infertile spermatozoa showed characteristics commensurate with a volume regulatory lesion (bent tails and poor flagellar vigour). The abnormality was observed even within the cauda epididymidis, but was exacerbated in the standard 'physiological' medium in which wild-type spermatozoa showed normal characteristics. The 'knockout' spermatozoa were apparently unable to enter the oviduct after mating in vivo (Yeung et al., 2000) and it was deduced that their resultant infertility was a consequence of their compromised motility. Regulatory volume decrease may also be an important factor with respect to sperm survival during or after cryopreservation. Major local osmotic gradients across sperm membranes are generated during the freezing and thawing cycle. However, studies by Gao et al. (1993) and Holt and North (1994) have shown that most cell death occurs during thawing, at a time when the dehydrated spermatozoa would be exposed to severely hypo-osmotic conditions (see Gilmore et al., 1996).

During preliminary studies on the volume response of boar spermatozoa to hypo-osmotic stress, we observed regulatory volume decrease behaviour that differed from that reported for bull spermatozoa by Kulkarni et al. (1997). Therefore, in the present study regulatory volume decrease in boar spermatozoa was compared and contrasted with that in bull spermatozoa, by investigating both the time course of the process and its modulation by quinine $\left(\mathrm{K}^{+}\right.$ channel blocker) and valinomycin ( $\mathrm{K}^{+}$ionophore). The possible involvement of the sodium-potassium pump and the $\mathrm{K}^{+}-\mathrm{Cl}^{-}$cotransporter in boar sperm regulatory volume decrease was also tested. Parallel studies on bull spermatozoa were performed to obtain a direct comparison, as our basic experimental protocol differed significantly from that of Kulkarni et al. (1997).

\section{Materials and Methods}

In essence, our experimental approach consisted of diluting washed sperm samples into iso-osmotic (300 mosmol kg-1) or hypo-osmotic (180 mosmol kg-1) medium at $39^{\circ} \mathrm{C}$, and taking sub-samples at timed intervals thereafter for analysis of the population volume distributions. Effects were largely judged by comparing the modal volumes of such distributions. Much of the methodology used was based on an earlier study of sperm volumetric behaviour (Petrounkina et al., 2000).

\section{Materials}

Chemicals were obtained from Merck AG (Darmstadt) and Sigma-Aldrich (Steinheim) unless otherwise stated.

\section{Basal experimental medium}

Two variants of a standard Hepes-buffered saline medium (HBSM: Harrison et al., 1993) were used as the vehicles for experimentation. Iso-osmotic HBSM (isoHBSM;

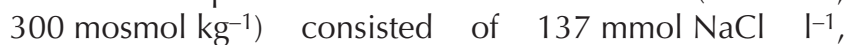
$10 \mathrm{mmol}$ glucose $\mathrm{I}^{-1}, 2.5 \mathrm{mmol} \mathrm{KOH} \mathrm{\textrm {I } ^ { - 1 }}$ and $20 \mathrm{mmol}$ Hepes $\mathrm{I}^{-1}$ buffered with $\mathrm{NaOH}$ to $\mathrm{pH} 7.4$ at $39^{\circ} \mathrm{C}$. Hypoosmotic HBSM (hypoHBSM; 180 mosmol kg-1) was prepared by diluting isoHBSM with water to the correct osmolality. The media were passed through a $0.2 \mu \mathrm{m}$ filter before use to minimize detection of particulate 'noise' during cell volume measurements.

\section{Semen processing}

Bull semen was obtained from four fertile Holstein animals from the Institute of Reproductive Medicine's bull colony. The samples were collected (generally twice a week) using an artificial vagina. Immediately after collection, the semen was transferred to the laboratory and diluted in a simple Tris-citrate-fructose extender (240

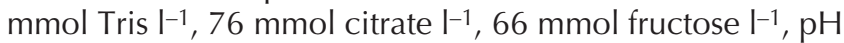
$7.5,300 \mathrm{mOsm} \mathrm{kg}^{-1}$; c.f. Foote, 1970) to a concentration of about $3 \times 10^{8}$ cells $\mathrm{ml}^{-1}$. Aliquants $(4 \mathrm{ml})$ were washed through a two-step gradient of $35 \%$ and $70 \%(\mathrm{v} / \mathrm{v})$ isoosmotic Percoll-saline (Harrison et al., 1993). After removal of the supernatant layers by aspiration, the loose sperm pellet was resuspended in residual 70\% (v/v) Percoll (about 1 $\mathrm{ml}$ ) and then diluted in isoHBSM containing $10 \mathrm{mg} \mathrm{BSA} \mathrm{ml}^{-1}$ to a final sperm concentration of about $2 \times 10^{8}$ cells $\mathrm{ml}^{-1}$.

Boar semen ('sperm-rich fraction') was obtained from ten fertile animals of either purebred or crossbred Pietrain breed, from the Institute of Reproductive Medicine's boar colony. The samples were collected (generally twice a week) by the 'gloved hand' method via sterile gauze (to remove gel). Immediately after collection the semen was transferred to the laboratory and diluted in BTS extender (Johnson et al., 1988) to a concentration of $0.8-1.0 \times 10^{8}$ cells $\mathrm{ml}^{-1}$. Aliquants $(3 \mathrm{ml})$ were washed through Percoll as described above for bull spermatozoa, after which the sperm pellet was resuspended in residual $70 \%$ $(\mathrm{v} / \mathrm{v})$ Percoll to a final concentration of about $2 \times 10^{8}$ cells $\mathrm{ml}^{-1}$.

Before incubation, semen samples were maintained at a minimum of $25^{\circ} \mathrm{C}$ to avoid cold shock. Processed samples were used for the experimental studies within $1 \mathrm{~h}$ of collection. 


\section{Experimental protocol}

Investigation of time course of volume changes in bull spermatozoa. Aliquants of washed and diluted sperm suspensions were diluted into $8 \mathrm{ml}$ volumes of isoHBSM at $39^{\circ} \mathrm{C}$ (final sperm cell concentration $0.5-1.0 \times 10^{7} \mathrm{ml}^{-1}$ ); BSA ( $1 \mathrm{mg} \mathrm{ml}^{-1}$ ) was included to maintain viability and prevent adhesion to surfaces (see Harrison et al., 1978, 1982). After $10 \mathrm{~min}$ preincubation, $100 \mu \mathrm{l}$ samples were transferred to $8 \mathrm{ml}$ of either hypoHBSM-BSA or isoHBSMBSA and incubated further at $39^{\circ} \mathrm{C}$. The iso-osmotic sperm suspension was sampled for cell volume measurement after $2 \mathrm{~min}$, whereas the hypo-osmotic suspension was sampled after $30 \mathrm{~s}$ and 2, 5, 10 and $20 \mathrm{~min}$.

Investigation of time course of volume changes in boar spermatozoa. Aliquants of washed sperm suspensions were diluted and preincubated in isoHBSM before dilution into either hypoHBSM or isoHBSM as described above for bull spermatozoa (final sperm concentration approximately $2.5 \times 10^{5} \mathrm{ml}^{-1}$ ); BSA was not included in the media for boar spermatozoa as viability was maintained in its absence. The iso-osmotic sperm suspension was sampled for cell volume measurement after $2 \mathrm{~min}$, whereas the hypoosmotic suspension was sampled after 2, 5, 10 and $20 \mathrm{~min}$.

Initial experiments were performed on both sperm species to demonstrate that volume distributions and modal volumes remained essentially constant during $20 \mathrm{~min}$ incubation in isoHBSM at $39^{\circ} \mathrm{C}$.

Investigation of quinine action in bull spermatozoa. Aliquants of washed sperm suspensions were diluted at $39^{\circ} \mathrm{C}$ into $8 \mathrm{ml}$ volumes of isoHBSM containing $1 \mathrm{mg} \mathrm{BSA} \mathrm{ml}^{-1}$ and $1 \mathrm{mmol}$ quinine $\mathrm{I}^{-1}$ (final sperm cell concentration 0.5$1.0 \times 10^{7} \mathrm{ml}^{-1}$ ). The suspension was then incubated for 10 min at $39^{\circ} \mathrm{C}$. A control sample was similarly incubated in isoHBSM-BSA without additive. After 10 min preincubation, $100 \mu \mathrm{l}$ samples from the quinine-containing suspension were transferred to $8 \mathrm{ml}$ of either hypoHBSM-BSA or isoHBSM-BSA at $39^{\circ} \mathrm{C}$, each containing either quinine or quinine plus $1 \mu \mathrm{mol}$ valinomycin $\mathrm{I}^{-1}$; the control sample was diluted in the absence of these additives. (The choice of these concentrations of quinine and valinomycin enabled a direct comparison with the experiments of Kulkarni et al. (1997)). The dilute suspensions were then incubated further at $39^{\circ} \mathrm{C}$. The iso-osmotic sperm suspensions were sampled for cell volume measurement after $2 \mathrm{~min}$, whereas the hypo-osmotic suspensions were sampled after $30 \mathrm{~s}$ and 2, 5, 10 and $20 \mathrm{~min}$.

Investigation of modulators of ion transport in boar spermatozoa. Aliquants of washed sperm suspensions were preincubated in HBSM as described for bull spermatozoa in the presence of various concentrations of quinine, ouabain and butylindazone (Alexis $\mathrm{GmbH}$, Grünberg); sperm concentration during this preincubation was $0.5-1.0 \times 10^{7}$ cells $\mathrm{ml}^{-1}$. Control samples were similarly incubated in isoHBSM without additives.
After the $10 \mathrm{~min}$ preincubation, samples from each suspension were transferred to HBS media each containing the same additive as the parent preincubation medium (final sperm concentration about $1 \times 10^{5} \mathrm{cells} \mathrm{ml}^{-1}$ ) and incubated as described above for bull spermatozoa. The iso-osmotic sperm suspensions were sampled for cell volume measurement after $2 \mathrm{~min}$, whereas the hypoosmotic suspensions were sampled after 2, 5, 10 and 20 min.

The effects of ionophores on quinine treatment were tested by including $1 \mu \mathrm{mol}$ valinomycin $\mathrm{I}^{-1}$ or $100 \mathrm{nmol}$ gramicidin $\mathrm{I}^{-1}$ in the medium into which the spermatozoa were diluted after preincubation. (From preliminary tests using a range of gramicidin concentrations, $100 \mathrm{nmol} \mathrm{I}^{-1}$ was chosen to ensure that most of the cells remained viable (unstained by propidium iodide, see below) during incubation; this concentration is known to be effective in other cell types.) In control experiments, spermatozoa were preincubated with the ionophores for $10 \mathrm{~min}$ at $39^{\circ} \mathrm{C}$ in the absence of quinine, before dilution into isoHBSM or hypoHBSM containing the same concentration of ionophore.

In all the experiments involving ion transport modulators, where an additive was added from a stock solution prepared in alcohol or dimethylsulphoxide (DMSO), the final concentration of solvent in the testing solution was $<0.5 \%(\mathrm{v} / \mathrm{v})$.

\section{Sperm quality estimations}

Sperm morphology was examined in samples taken from the native ejaculates shortly after semen collection and estimations were made of motility and acrosomal integrity at the start of each experiment. The methodology used for these assessments was as described by Petrounkina et al. (2000) and references therein. Cell viability was checked during the experimental incubations using propidium iodide staining (Harrison and Vickers, 1990). In preliminary studies, no significant loss of viability of boar spermatozoa was observed during $20 \mathrm{~min}$ incubation in either iso- or hypo-HBSM. Therefore, unless otherwise stated, the boar samples were assessed routinely in wet mounts after 5 min incubation in both iso- and hypo-osmotic conditions. For bull sperm suspensions, samples (routinely taken at $5 \mathrm{~min}$ ) were loaded with propidium iodide as for boar sperm suspensions, but the assessments of the bull samples were carried out on air-dried unfixed smears to avoid artefactual sperm deterioration that tended to occur during observation of wet mounts. Under these conditions, 'dead' spermatozoa had evenly bright fluorescent heads, whereas 'live' spermatozoa were only slightly fluorescent (in the apical region of the head).

\section{Cell volume measurement}

At each sampling time-point, a single sample from each incubated sperm suspension was passed through a CASY 1 cell counter (Schaerfe Systems GmbH, Reutlingen), which produced cell volume information on the basis of cell 
frequency distribution within 1024 electronic 'volume' channels. The sample volume setting was $200 \mu \mathrm{l}$ and the size scale $10 \mu \mathrm{m}$; each sampling obtained data from $>1.2 \times 10^{4}$ cells.

As the electrical conductivity of hypoHBSM was lower than that of isoHBSM (different concentrations of electrolyte), a correction factor (1.10) was applied to data recorded from sperm suspensions in hypoHBSM. The correction factor was obtained according to Petrounkina et al. (2000).

\section{Analysis of volumetric data}

Unless otherwise stated, the analyses used the modal values (corrected for the different osmotic conditions) of the volume distribution; these values were obtained directly from the cell volume measurements using the CASY software. The modal volume has been shown to be a more sensitive parameter of volume change than mean volume (Petrunkina and Töpfer-Petersen, 2000).

The relative volume shift $V_{\mathrm{r}}$ was used as a measure of the volume regulation in response to aniso-osmotic conditions. It was defined as $V_{\mathrm{r}}=V_{\text {aniso }} / V_{\text {iso, }}$ where $V_{\text {aniso }}$ was the modal value of the aniso-osmotic volume distribution and $V_{\text {iso }}$ was the modal value of the iso-osmotic volume distribution. When several sperm subpopulations contributed to a distribution, the values pertaining to the largest osmotically active subpopulation were used. A cell subpopulation was considered osmotically active if its $V_{r}$ was $>1$.

The observed effects of modulators of ion transport were verified by analysis of variance (General Linear Model; SAS Software, SAS Institute Inc. Cary, NC). The KolmogorovSmirnoff two-sample test was used to analyse the effects with respect to the shape of the volume distribution curves (Petrounkina et al., 2000). The distribution analysis was carried out on the exported numerical CASY data (the count number in each of the 1024 effective particle size channels). Each data set was 'normalized' by the mathematical procedure described by Petrounkina et al. (2000) to allow proper statistical comparisons, and pair-wise comparisons were made on the normalized distribution functions.

Non-linear modelling (Procedure NLIN; SAS Software) was used to estimate the $\mathrm{IC}_{50}$ value of quinine treatment as well as the half-times of cell shrinkage. Model functions were fitted empirically to the experimental data, and first derivatives were obtained from these by partial differentiation. The functions and their first derivatives were entered into the computer program, which calculated the parameters by an iterative procedure.

Unless otherwise stated, values presented are mean \pm SEM. Differences were considered significant at $P<0.05$.

\section{Results}

\section{Conventional parameters}

As judged by conventional sperm parameters, the native ejaculates of the boars and bulls involved in the study were in the normal range, although there were sample differences. Motility was in the range $70-90 \%$, morphological abnormalities were $<30 \%$, the viability of the boar spermatozoa was $>80 \%$ and the viability of the bull spermatozoa was $>75 \%$. Massal motility ('wave motion') of the bull semen was at least of medium intensity (score of at least 2 on a scale of $0-4$ ).

\section{Cell volume in boar and bull sperm samples during incubation under iso-osmotic conditions}

No changes in cell volume were observed during incubation of either bull or boar spermatozoa in isoHBSM (300 mosmol $\mathrm{kg}^{-1}$ ) at $39^{\circ} \mathrm{C}$ over a 20 min period. The modal volume after $20 \mathrm{~min}$ relative to that after $2 \mathrm{~min}$ was $1.00 \pm 0.02$ in boar spermatozoa and $1.00 \pm 0.01$ in bull spermatozoa $(n=3)$. There were no significant differences between the shapes of cell volume distributions at different sampling points for any of the samples in either species.

Iso-osmotic incubation in the presence of $1 \mathrm{mmol}$ quinine $\mathrm{I}^{-1}$ did not affect the cell volume of either bull or boar spermatozoa during $20 \mathrm{~min}$ incubation at $39^{\circ} \mathrm{C}$. The modal volume after $20 \mathrm{~min}$ of incubation relative to that after 2 min was $1.00 \pm 0.03$ in boar spermatozoa and $1.00 \pm 0.02$ in bull spermatozoa $(n=3)$. Again, no significant differences between cell volume distribution shapes were seen at the different sampling points for any of the samples.

\section{Cell volume changes in boar and bull spermatozoa in response to hypo-osmotic conditions}

Time course in bull spermatozoa. Sperm samples from eight bull ejaculates were exposed to hypoHBSM (180 mosmol $\mathrm{kg}^{-1}$ ) for $30 \mathrm{~s}, 2,5,10$ and $20 \mathrm{~min}$. The mean population modal volumes relative to those in isoHBSM $\left(V_{r}\right)$ are shown (Fig. 1a). Swelling, already clearly advanced after $30 \mathrm{~s}$ exposure, reached a maximum at $5 \mathrm{~min}$ and then decreased thereafter; however, after $20 \mathrm{~min}$, the modal volume was still significantly above the iso-osmotic (control) value. Although there were large individual variations with respect to the degree of swelling (see below), the mean $V_{r}$ values at $30 \mathrm{~s}$ and $2 \mathrm{~min}$ were significantly different from those at $20 \mathrm{~min}$, and the difference between the mean $V_{r}$ values at $5 \mathrm{~min}$ and at $20 \mathrm{~min}$ was 'noteworthy', although not significant $(P<0.09)$.

Individual bull ejaculates differed considerably with respect both to the time course and the degree of regulatory volume decrease. In four of eight samples, there were significant differences between the volume distribution curves at $30 \mathrm{~s}$ and those at $20 \mathrm{~min}$, while in a further three samples, it was the 5 min curves that were significantly different from the 20 min curves.

For two of four bulls, there were no differences between two different ejaculates of the same bull with respect to the volume distribution curve, whereas for the other two bulls, the two ejaculates were significantly different. 
(a)

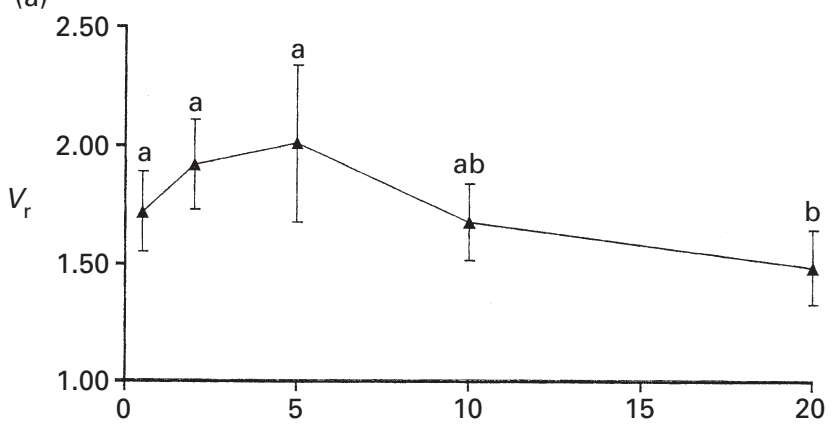

(b)

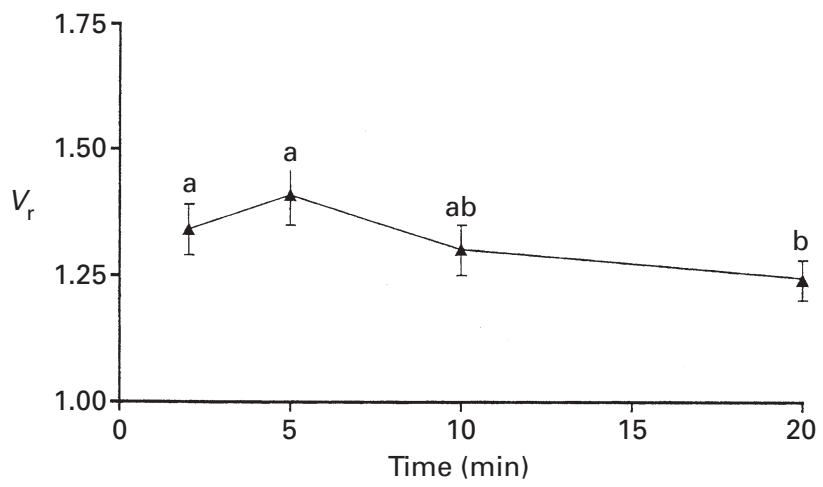

Fig. 1. Time course of volume response of (a) bull and (b) boar spermatozoa to hypo-osmotic stress at $39^{\circ} \mathrm{C}$. Washed spermatozoa, preincubated in iso-osmotic Hepes-buffered saline medium (isoHBSM) were diluted into either hypo-osmotic HBSM (hypoHBSM) or isoHBSM and incubated further. Volume measurements were made at various times after dilution. $V_{\mathrm{r}}$ is the modal hypo-osmotic volume at the given time point relative to the modal iso-osmotic volume after 2 min (mean \pm SEM; bull: $n=8$; boar: $n=20$ ). abValues that do not share a common letter are significantly different $(P<0.05)$.

Time course in boar spermatozoa. Boar spermatozoa were exposed to hypoHBSM for 2, 5, 10 and $20 \mathrm{~min}$. Twenty ejaculates were tested. The mean population modal volumes relative to those in isoHBSM $\left(V_{\mathrm{r}}\right)$ are shown (Fig. 1b). Swelling, already clearly advanced after $2 \mathrm{~min}$ exposure, reached a maximum at $5 \mathrm{~min}$ and then decreased thereafter; however, after $20 \mathrm{~min}$, the modal volume was still significantly above the control (iso-osmotic) value.

Differences between individual boar ejaculates with respect to the time course and the degree of regulatory volume decrease were much less than in bulls (compare Fig. 1 b with Fig. 1a). In 15 of 20 ejaculates, there was a significant difference between the volume distribution curve after $2 \mathrm{~min}$ and that after $20 \mathrm{~min}$. For two further ejaculates, it was the 5 min value that differed significantly from the 20 min value. Comparison of the 2 min distribution curves of two further ejaculates from each of four boars showed no significant within-boar differences. However, the volume distribution curve shapes of the hypoosmotically-treated samples differed among the four boars.
Two animals gave similar curves, whereas each of the other two boars had volume distribution curves with shapes that differed significantly from the others.

\section{Effect of quinine on bull sperm volume regulation}

Incubation of bull sperm suspensions with $1 \mathrm{mmol}$ quinine $\mathrm{I}^{-1}$, a well-established inhibitor of $\mathrm{K}^{+}$channels (see Sarkadi and Parker, 1991), did not affect iso-osmotic cell volume but very strongly affected volume response to hypoosmotic stress. After only $30 \mathrm{~s}$ exposure to hypo-osmotic conditions, quinine-treated bull spermatozoa had already swollen much more than untreated samples (Fig. 2a). Swelling of the quinine-treated samples was maximal at $30 \mathrm{~s}$ and, thereafter, decreased steadily with time, until by 20 min the modal volume was essentially the same as that of untreated samples. There was no significant effect of quinine on cell viability after 5 min incubation (mean values of $22 \%$ and $28 \%$ of propidium iodide-positive cells under iso- and hypo-osmotic conditions, respectively, compared with $21 \%$ and $23 \%$, respectively, in the absence of quinine). Under iso-osmotic conditions, viability in the presence of quinine remained similarly high during $20 \mathrm{~min}$ incubation. However, under hypo-osmotic conditions, the percentage of propidium iodide-positive cells after $20 \mathrm{~min}$ in the presence of quinine was $41 \%$ compared with a control value of $27 \%$.

In terms of variations in response, in five of eight bull ejaculates the volume distributions of the quinine-treated samples after $30 \mathrm{~s}$ of incubation differed significantly from the untreated samples (Fig. 3a). Those ejaculates that did not respond strongly to quinine treatment showed either a very high or a very low response to hypo-osmotic shock and, in the absence of quinine, showed no regulatory volume decrease.

When bull spermatozoa were diluted in the presence of the $\mathrm{K}^{+}$-specific ionophore valinomycin $\left(1 \mu \mathrm{mol} \mathrm{I}^{-1}\right)$ after pretreatment with 1 mmol quinine $\mathrm{I}^{-1}$, initial swelling (after 30 s hypo-osmotic exposure) was almost the same as for control samples (no quinine or valinomycin), and much less than the swelling shown by samples treated with quinine only (see Fig. 2a). During further incubation under hypoosmotic conditions, the volumes of the bull spermatozoa treated with quinine and valinomycin together decreased rapidly to values close to the initial iso-osmotic volume. Regulatory volume decrease half-times for quinine-treated and quinine plus valinomycin-treated spermatozoa were estimated to be 9.0 and $2.5 \mathrm{~min}$, respectively. (The later observed changes in cell volume may have been largely due to ongoing cell death: $>50 \%$ of cells were dead after 5 min exposure to quinine plus valinomycin.)

\section{Effect of quinine on boar sperm volume regulation}

As with bull spermatozoa, incubation of boar sperm suspensions with $1 \mathrm{mmol}$ quinine $\mathrm{I}^{-1}$ did not affect isoosmotic cell volume but strongly affected volume response to hypo-osmotic stress, increasing swelling compared with 

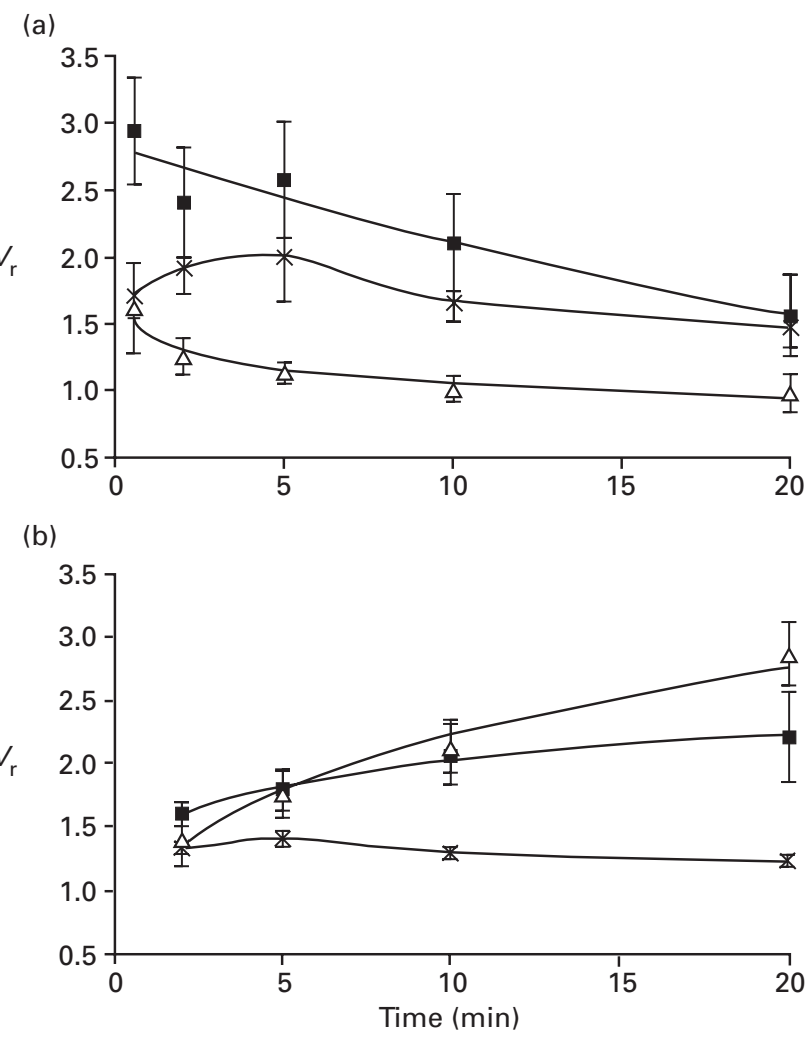

Fig. 2. Effects of quinine and valinomycin on the volume responses of (a) bull and (b) boar spermatozoa to hypo-osmotic stress at $39^{\circ} \mathrm{C}$. Spermatozoa were preincubated in iso-osmotic Hepes-buffered saline medium (isoHBSM) in the presence or absence of $1 \mathrm{mmol}$ quinine $\mathrm{I}^{-1}$. Samples pretreated with quinine were then diluted into either hypo-osmotic HBSM (hypoHBSM) or isoHBSM containing the same concentration of quinine (ם), or into the same media supplemented with $1 \mu \mathrm{mol}$ valinomycin $\mathrm{I}^{-1}(\triangle)$. Samples incubated in the absence of quinine (control; $X$ ) were diluted into hypo- or isoHBSM without additives. Cell volume measurements $\left(V_{\mathrm{r}}\right)$ were made at various intervals after dilution.

untreated samples (Figs $2 \mathrm{~b}$ and $3 \mathrm{~b}$ ). However, the time course of the volume changes in quinine-treated boar spermatozoa was markedly different from that in similarly treated bull spermatozoa: in boar spermatozoa the increased swelling developed over a relatively long time period (compare Fig. 2b with Fig. 2a). After 2 min of exposure to hypo-osmotic conditions, there were differences between the volume distributions of quininetreated and untreated spermatozoa in four of seven samples of boar spermatozoa. After $5 \mathrm{~min}$ exposure, volume distribution curves of six of seven treated samples had shifted significantly to higher values; after $20 \mathrm{~min}$, a treatment difference was found for all seven samples. The degree of the effect differed among ejaculates: two of seven samples showed moderate or low volume increase due to quinine treatment, whereas the other five showed a very high increase. The two samples in which quinine treatment had a low effect showed strong response to hypo-osmotic conditions in the absence of quinine $\left(V_{r}=1.89\right.$ and $V_{r}=$
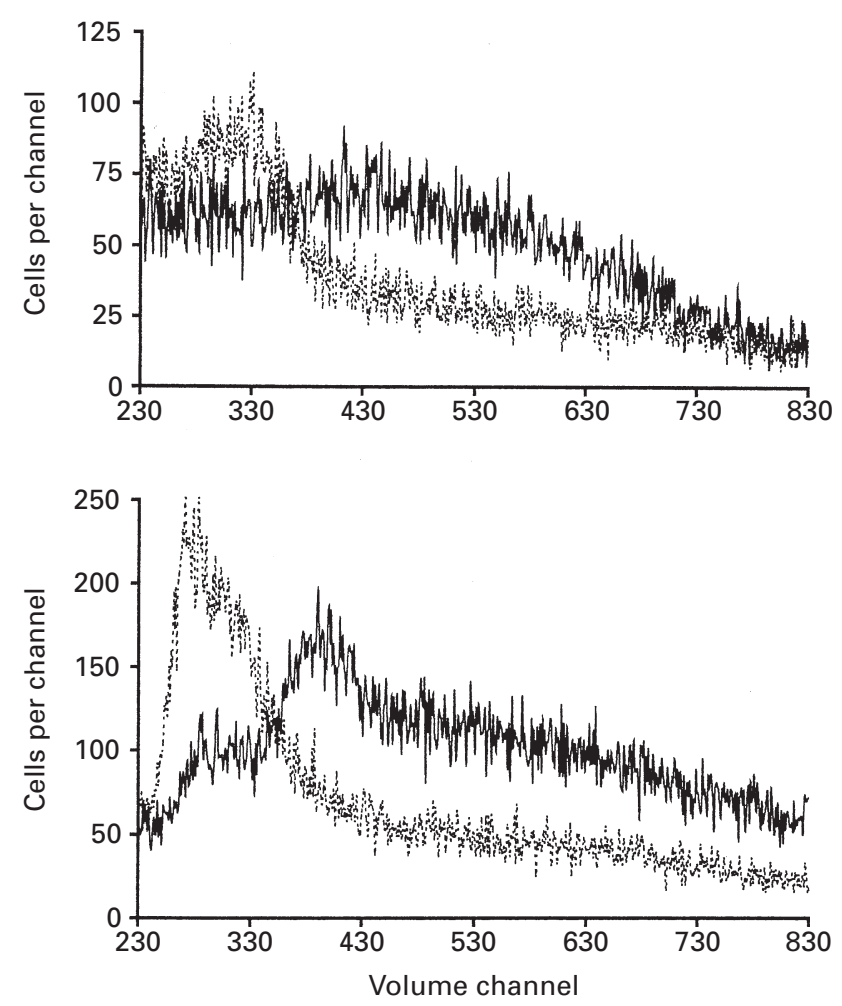

Fig. 3. Effect of quinine on the volume distribution of (a) bull and (b) boar sperm populations exposed to hypo-osmotic conditions at $39^{\circ} \mathrm{C}$. Sperm samples were preincubated in iso-osmotic Hepesbuffered saline medium (isoHBSM) in the presence or absence of quinine $\left(1 \mathrm{mmol} \mathrm{I}^{-1}\right)$, diluted into hypo-osmotic HBSM (hypoHBSM) with or without quinine, respectively, and incubated for a further $30 \mathrm{~s}$ (bull) or $20 \mathrm{~min}$ (boar) before cell volume measurements $\left(V_{\mathrm{r}}\right)$ were made. The distribution curves shown are representative treatment pairs from particular experiments (that is, from single ejaculates). Broken line (----): control treatment; solid line $(\stackrel{-}{-})$ : treatment with quinine. Volume distributions were significantly different between control and quinine-treated groups $(P<0.05$, Kolmogorov-Smirnoff test $)$.

2.54 at 2 min, which are much higher than the mean value $V_{r}=1.34$ at 2 min shown in Fig. 1b) and also good regulatory volume decreases $\left(V_{r}=1.00\right.$ and 1.65, respectively, at $20 \mathrm{~min})$. For four sperm samples showing normal hypo-osmotic responses $\left(V_{\mathrm{r}}=1.40 \pm 0.07\right.$ at $5 \mathrm{~min}$ and $1.25 \pm 0.05$ at $20 \mathrm{~min}$ ), pretreatment with quinine increased $V_{r}$ to $1.61 \pm 0.20$ after $5 \mathrm{~min}$ hypo-osmotic exposure and to $2.97 \pm 0.21$ after $20 \mathrm{~min}$.

The effect of quinine pretreatment could be shown to be dose-dependent. However, the dose-effect curve with respect to $5 \mathrm{~min}$ exposure to hypo-osmotic conditions (Fig. 4a) differed markedly from that with respect to $20 \mathrm{~min}$ exposure (Fig. $4 \mathrm{~b}$ ); the $\mathrm{IC}_{50}$ value was estimated to be 1.18 $\mathrm{mmol} \mathrm{I}^{-1}$ for the 5 min effects and $0.82 \mathrm{mmol} \mathrm{I}^{-1}$ for the 20 min effects. Boar sperm cells remained essentially viable in quinine concentrations up to $1 \mathrm{mmol} \mathrm{I}^{-1}$; at no time points were there significantly $>10 \%$ propidium iodide-positive cells (mean values, $n=4$ ). Only after 20 min of incubation 
(a)

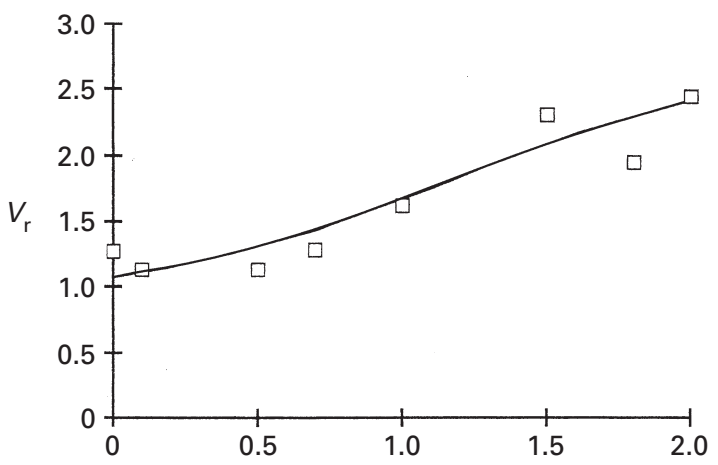

(b)

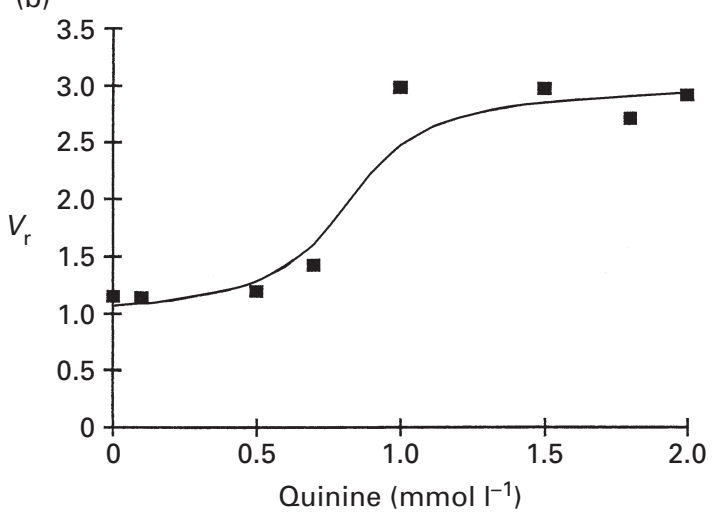

Fig. 4. Dose dependency of quinine treatment. Boar spermatozoa were preincubated in iso-osmotic Hepes-buffered saline medium (isoHBSM) containing various concentrations of quinine and then diluted into either hypo-osmotic HBSM (hypoHBSM) or isoHBSM containing the same concentrations of quinine as during preincubation. Cell volume measurements $\left(V_{r}\right)$ were made on samples in isoHBSM after 2 min and on samples in hypoHBSM after (a) 5 and (b) $20 \mathrm{~min}$. The data relating to four independent experiments (four different boars) are presented in terms of mean $V_{r}$.

with 2 mmol quinine $\mathrm{I}^{-1}$ did the percentage of propidium iodide-positive cells increase, to mean values of $30 \%$ and $35 \%$ under iso-osmotic and hypo-osmotic conditions, respectively, compared with control values of $7 \%$ and $9 \%$, respectively.

When boar spermatozoa were pretreated with $1 \mathrm{mmol}$ quinine $\mathrm{I}^{-1}$ and diluted in the presence of $1 \mu \mathrm{mol}$ valinomycin $\mathrm{I}^{-1}$, surprising results were obtained. Initial swelling after 2 min hypo-osmotic exposure was essentially the same as for control samples (no quinine or valinomycin), whereas samples treated with quinine only swelled more than control samples. However, during further incubation under hypo-osmotic conditions, unlike bull spermatozoa, boar spermatozoa treated with quinine and valinomycin together continued to swell, and by 20 min had swollen considerably more than spermatozoa treated with quinine only. For quinine plus valinomycintreated samples, $V_{\mathrm{r}}$ reached $1.76 \pm 0.16$ after $5 \mathrm{~min}$ and $2.87 \pm 0.21$ after $20 \mathrm{~min}$, whereas the respective values for quinine-treated spermatozoa were $V_{r}=1.80 \pm 0.14$ and
$V_{r}=2.21 \pm 0.30$, respectively (Fig. 2 b). The swelling halftime (time to $50 \%$ maximum volume) for quinine-pretreated spermatozoa was estimated to be $7 \mathrm{~min}$, whereas that for valinomycin-treated quinine-pretreated spermatozoa was 9.5 min. Valinomycin caused slight decreases in the viability of quinine-treated spermatozoa: in terms of propidium iodide-positive cells, under iso- and hypoosmotic conditions, respectively, mean values of $12 \%$ and $14 \%$ after $5 \mathrm{~min}$, and $26 \%$ and $33 \%$ after $20 \mathrm{~min}$ were obtained, compared with $10 \%$ and $10 \%$ after 5 min, and $15 \%$ and $15 \%$ after $20 \mathrm{~min}$ in the absence of valinomycin.

Tests on boar spermatozoa from four independent ejaculates showed that treatment with valinomycin only induced a slight shift of the iso-osmotic volume distribution curve to higher values, increasing the modal volume to $118 \%$ compared with the control sample. Valinomycin also affected the hypo-osmotic volume distribution curves, increasing both the modal volume and $V_{r}$ slightly (after 10 min exposure to the hypo-osmotic conditions, $V_{\mathrm{r}}=1.56$ for the treated samples compared with $V_{\mathrm{r}}=1.29$ for untreated samples). However, during further incubation to $20 \mathrm{~min}$, swelling neither increased further nor decreased. Treatment with valinomycin only caused a slight increase in the percentage of propidium iodide-positive cells: under isoand hypo-osmotic conditions respectively, mean values of $22 \%$ and $27 \%$ after $5 \mathrm{~min}$ and $27 \%$ and $29 \%$ after $20 \mathrm{~min}$, compared with $14 \%$ and $16 \%$ after 5 min and $15 \%$ and $23 \%$ after $20 \mathrm{~min}$ in the absence of valinomycin.

\section{Investigation of possible involvement of other $\mathrm{K}^{+}$-related transport systems in boar sperm volume regulation}

In four tests (ejaculates from different boars), ouabain, a well-known inhibitor of the plasma membrane $\mathrm{Na}^{+}-\mathrm{K}^{+}-$ transporting ATPase (for example, Cheung et al., 1982), had no significant effect either on iso-osmotic volume or on hypoosmotically induced volume changes in boar spermatozoa at $100 \mu \mathrm{mol} \mathrm{I}^{-1}$. $1 \mathrm{mmol}$ ouabain $\mathrm{I}^{-1}$ induced a significant increase in the iso-osmotic volume (to $123 \%$ of the control value), but no other volumetric parameter was affected. No effects on viability were observed in this concentration range.

DIOA, an inhibitor of $\mathrm{K}^{+}-\mathrm{Cl}^{-}$co-transport (Garay et al., 1988; Arrazola et al., 1993), had no significant effect on any volumetric parameters in boar spermatozoa when tested in the range $25-250 \mu \mathrm{mol} \mathrm{I-1} \quad(n=3-4$ ejaculates from different boars). DIOA treatment had no noteworthy effects on viability below $125 \mu \mathrm{mol} \mathrm{^{-1 }}$. However, after $20 \mathrm{~min}$ incubation in the presence of $250 \mu \mathrm{mol}$ DIOA I-1, the percentages of propidium iodide-positive cells had increased to means of $28 \%$ and $42 \%$ under iso- and hypoosmotic conditions, respectively, compared with control (untreated) values of $15 \%$ and $18 \%$.

\section{Effect of gramicidin on volume regulation and reversibility of the quinine effect in boar spermatozoa}

The inability of the $\mathrm{K}^{+}$-specific ionophore valinomycin to reverse the blocking effect of quinine on boar sperm 
regulatory volume decrease led us to test the effect of the non-selective monovalent cation ionophore gramicidin. The experiment was performed on six boar ejaculates. All samples swelled strongly in response to hypo-osmotic conditions $\left(V_{r}\right.$ value at $5 \mathrm{~min}$ was approximately 1.9 compared with the 'normal mean' value of about 1.4; compare with Fig. 1b). However, while three of the ejaculates showed a normal regulatory volume decrease, the other three showed none during $20 \mathrm{~min}$ exposure; in these samples, cell volumes continued to increase. Moreover, treatment with quinine caused relatively little extra hypo-osmotic swelling in the samples that possessed regulatory volume decrease ability, whereas the samples without regulatory volume decrease ability swelled considerably more. As the two groups responded differently to gramicidin, their results are presented separately: samples with normal regulatory volume decrease ('good' regulators; Fig. 5a) and samples that failed to show regulatory volume decrease ('poor' regulators; Fig. 5b).

In both groups, the inclusion of $100 \mathrm{nmol}$ gramicidin $\mathrm{I}^{-1}$ (a concentration at which spermatozoa remained viable) enhanced hypo-osmotic swelling in quinine-treated spermatozoa. However, 'good' regulators underwent enhanced regulatory volume decrease after treatment with gramicidin only, whereas the ionophore was unable to restore regulatory volume decrease in the 'poor' regulators.

\section{Discussion}

There are three essential findings of our investigations. Both boar and bull spermatozoa are able to reduce swelling caused by hypo-osmotic stress on a time-dependent basis (they show regulatory volume decrease, a mechanism present in somatic cell types but as yet poorly defined in sperm cells). In both species, the regulatory volume decrease is mediated to a considerable degree via ion channels that can be inhibited with quinine. However, while the $\mathrm{K}^{+}$ionophore valinomycin is able to overcome quinine inhibition of regulatory volume decrease in bull spermatozoa, it exacerbates hypo-osmotic swelling in boar spermatozoa.

In a previous study of bull sperm regulatory volume decrease, Kulkarni et al. (1997) could not discern a time course for the process; they deduced it to be extremely rapid in this species, already complete within $30 \mathrm{~s}$. However, in the present study both bull and boar spermatozoa showed regulatory volume decrease with a similar and clearly discernible time course. After dilution in hypo-osmotic medium, swelling took place quickly but soon slowed to a maximum after about $5 \mathrm{~min}$, after which the volume of the cells decreased in a curvilinear fashion. Swelling tended to be considerably greater in bull than in boar spermatozoa $\left(V_{r}\right.$ at $5 \mathrm{~min}=2.01 \pm 0.33$ in bull spermatozoa compared with $1.41 \pm 0.06$ in boar spermatozoa), possibly because the plasma membrane water conductivity in bull spermatozoa is more than tenfold higher than in boar spermatozoa (Curry, 2000). The time course of sperm regulatory volume decrease was (a)

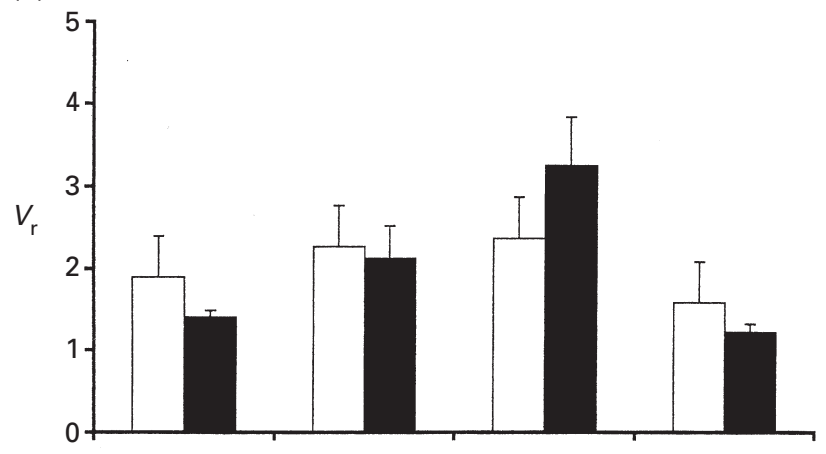

(b)

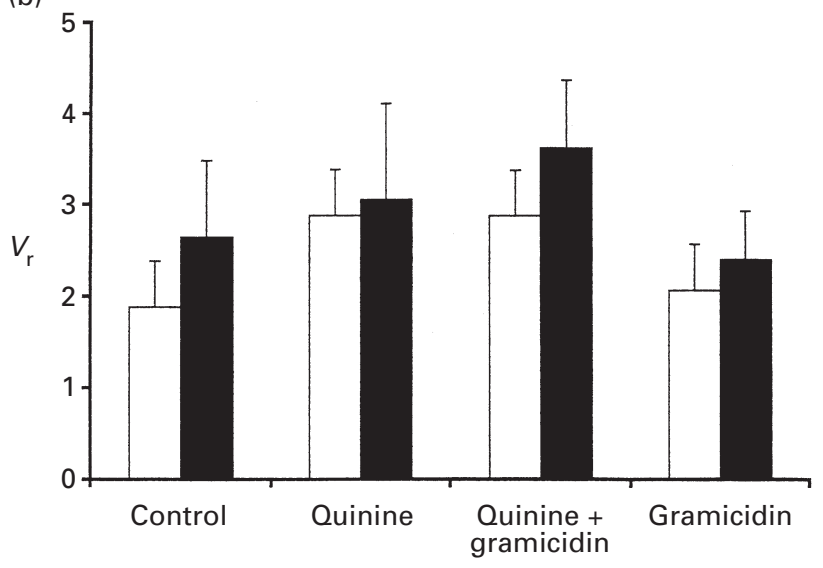

Fig. 5. Effect of gramicidin on volume regulation in boar spermatozoa: (a) 'good' volume regulators and (b) 'poor' volume regulators. Sperm samples were preincubated in iso-osmotic Hepes-buffered saline medium (isoHBSM) containing either 1 mmol quinine $\mathrm{I}^{-1}$ or $100 \mathrm{nmol}$ gramicidin $\mathrm{I}^{-1}$. Control samples were preincubated in isoHBSM without additives. After $10 \mathrm{~min}$, subsamples were diluted into either isoHBSM or hypo-osmotic HBSM (hypoHBSM) containing the same additives as the original suspensions; in the case of the quinine-treated spermatozoa, samples were also diluted into quinine-containing isoHBSM and hypoHBSM supplemented with $100 \mathrm{nmol}$ gramicidin $\mathrm{I}^{-1}$. Cell volume measurements were performed after $2 \mathrm{~min}$ in isoHBSM and after $5(\square)$ or $20 \mathrm{~min}(\square)$ in hypoHBSM.

relatively slow compared with that reported for lymphocytes by Cheung et al. (1982), in which swelling reached a maximum after about $1 \mathrm{~min}$ and had decreased to near the iso-osmotic volume by $10 \mathrm{~min}$.

Although several different ion transport mechanisms may play a role in volume regulation, in most mammalian cell types regulatory volume decrease is mediated primarily via a volume-activated opening of independent $\mathrm{K}^{+}$and $\mathrm{Cl}^{-}$ channels. As a cell swells in a hypo-osmotic environment, the channels are activated to allow these ions to exit the cell, thereby reducing internal osmolality and reversing swelling. It is important that both ions exit essentially in parallel to maintain electroneutrality during ion efflux. Thus, when quinine, a well-established inhibitor of swelling-activated $\mathrm{K}^{+}$ channels (see Sarkadi and Parker, 1991), is used to prevent 
efflux of $\mathrm{K}^{+}$, overall loss of electrolytes is essentially blocked, with the result that hypo-osmotic swelling is enhanced and little or no regulatory volume decrease occurs.

In the present study, regulatory volume decrease in both boar and bull spermatozoa was inhibited by treatment with quinine, although the time course of the resultant enhanced swelling was different in the two species. Quinine-treated boar spermatozoa continued to swell for at least $10 \mathrm{~min}$ after hypo-osmotic dilution, whereas in bull spermatozoa, swelling was already maximal by $30 \mathrm{~s}$ and decreased steadily thereafter (possibly continuing regulatory volume decrease). This latter finding conflicts somewhat with the findings of Kulkarni et al. (1997), who reported that $1 \mathrm{mmol}$ quinine $\mathrm{I}^{-1}$ inhibited bull sperm regulatory volume decrease completely during at least $10 \mathrm{~min}$. Although part of the decrease in bull sperm volume detected in the present study could have been due to increased bull sperm death in the presence of quinine (see Results section), most of the cells remained unstained with propidium iodide; moreover, our volumetric data related to the osmotically active subpopulation ( $V_{r}>1$; cells with 'intact' plasma membranes). As a result of the difference in the responses of bull and boar spermatozoa to quinine, the effects of DIOA and ouabain on boar sperm regulatory volume decrease were investigated to determine whether other $\mathrm{K}^{+}$transport mechanisms might be involved. Neither of these compounds had any effect. This finding was not unexpected, as the $\mathrm{K}^{+}-\mathrm{Cl}^{-}$co-transporter is of major importance in regulatory volume decrease in red blood cells only (Sarkadi and Parker, 1991; Al-Habori, 1994), and the ATP-dependent $\mathrm{Na}^{+-} \mathrm{K}^{+}$pump is considered to be of importance in regulating volume under iso-osmotic conditions (changes in relative ion concentrations) or hyperosmotic conditions (regulatory volume increase) only (Hoffmann and Dunham, 1995; O'Neill, 1999). Bredderman and Foote $(1971 \mathrm{a}, \mathrm{b})$ demonstrated an essential role for the $\mathrm{Na}^{+}-\mathrm{K}^{+}$pump in bull sperm volume maintenance under isoosmotic conditions. However, Rodriguez-Gil and Rigau (1996) were unable to detect any effect of ouabain on boar sperm swelling in response to hypo-osmotic shock, although they found an effect of ouabain on dog spermatozoa. The results of the present study, together with those of Kulkarni et al. (1997), indicate strongly that, as in many other animal cell types, swelling-activated $\mathrm{K}^{+}$channels play an important role in bull and boar sperm regulatory volume decrease; the results of Yeung et al. (1999) extend this deduction to mouse spermatozoa.

In many cell types, the $\mathrm{K}^{+}$-specific ionophore valinomycin is able to overcome quinine inhibition of regulatory volume decrease by providing an alternative exit for $\mathrm{K}^{+}$. In the present study, valinomycin restored regulatory volume decrease in hypo-osmotically stressed quinine-treated bull spermatozoa, as also reported by Kulkarni et al. (1997). However, in boar spermatozoa, valinomycin treatment exacerbated quinine-induced swelling markedly. The ability of valinomycin to overcome the quinine effect is dependent on there being a high $\mathrm{Cl}^{-}$conductivity with $\mathrm{K}^{+}$ conductivity the limiting factor. Should this condition not be fulfilled (when $\mathrm{Cl}^{-}$conductivity is low), valinomycin may have an opposite effect. The ionophore has been reported as inhibiting anion channels (Sarkadi et al., 1985) and, thus, its inhibition of a limited $\mathrm{Cl}^{-}$conductivity might have a greater effect than its enhancement of $\mathrm{K}^{+}$conductivity. Under these circumstances, valinomycin would increase quinine-induced swelling. Therefore, the paradoxical effect of valinomycin on quinine-treated boar spermatozoa can be interpreted as putative evidence that boar spermatozoa contain considerably fewer volume-sensitive $\mathrm{Cl}^{-}$channels than do bull spermatozoa. Volume-sensitive $\mathrm{Cl}^{-}$channels similar to those found in other cell types (Strange et al., 1996; Jentsch and Günther, 1997) have not so far been identified in spermatozoa, attention having been focussed on receptor-operated $\mathrm{Cl}^{-}$channels that appear to play a role in the acrosome reaction (Meizel, 1997). However, the studies of Yeung et al. (1999) provide strong evidence for volume-regulatory $\mathrm{Cl}^{-}$channels in mouse spermatozoa.

Given the apparent effect of volume regulatory lesions in mouse spermatozoa on fertility (Yeung et al., 2000), the large differences in hypo-osmotic response and in degree of regulatory volume decrease noted between individual animals in both species in the present study may be of physiological significance. Sperm samples from two of the boars whose response to gramicidin was tested were of particular interest. The spermatozoa from one boar showed good regulatory volume decrease in response to hypo-osmotic stress, whereas spermatozoa from the other showed none (spermatozoa continued to swell during $20 \mathrm{~min}$ ). Quinine almost abolished regulatory volume decrease in the 'good' responders and enhanced swelling considerably in the 'poor' responders. Treatment with the non-selective monovalent cation ionophore gramicidin had no effect on hypo-osmotic swelling in the 'poor' responders but it enhanced regulatory volume decrease in the 'good' responders. We interpret the latter result as suggesting that in the 'poor' responders the volume-sensitive $\mathrm{Cl}^{-}$channels are either few in number or the mechanism for activating them is faulty. The argument is as follows: loss of $\mathrm{K}^{+}$and $\mathrm{Cl}^{-}$as part of regulatory volume decrease must take place essentially under electroneutrality and, thus, can take place only if both $\mathrm{Cl}^{-}$and $\mathrm{K}^{+}$channels are activated together. If $\mathrm{Cl}^{-}$channels remain inactive or are relatively few in number compared with $\mathrm{K}^{+}$channels, little or no regulatory volume decrease can occur because electroneutrality cannot be maintained (c.f. 'poor' responders). In this case, quinine will cause increased swelling relative to its effect on cells with normal numbers of activated $\mathrm{Cl}^{-}$channels ('good' responders). If poor responders are treated with gramicidin before exposure to hypo-osmotic shock (to maximize cation efflux), exchange of ions remains limited because there are insufficient activated $\mathrm{Cl}^{-}$channels in these cells, and overall swelling takes place. However, when good responders are treated with gramicidin before hypoosmotic shock, regulatory volume decrease is enhanced because rapid net ion efflux can take place; electroneutrality is maintained due to the presence of many active $\mathrm{Cl}^{-}$channels.

It will clearly be of interest to investigate the molecular 
causes of such differences in regulatory volume decrease response further. Much remains to be elucidated. For example, no proof has yet been obtained that regulatory volume decrease in spermatozoa, as in most other mammalian cell types, is brought about by the actual activation of channels by the swelling process (Grinstein et al., 1984). The molecular sequence by which such activation might take place should also be investigated. A range of mechanisms have been proposed for other cell types (see Sarkadi and Parker, 1991; Parker, 1993; Hoffmann and Dunham, 1995), but as yet none have been defined. The sperm cell might present a useful model in which to study regulatory volume decrease because of its relative functional simplicity.

The authors would like to thank D. Waberski for organizing the collection of the samples of fresh bull semen for this study. A. M. Petrunkina was supported by the Deutsche Forschungsgemeinschaft (grant refs To114/3-3 and To114/3-4). M. Hebel was supported by the Dr. Dr. h.c. Karl Eibl-Foundation (Neustadt a.d. Aisch). R. A. P. Harrison was supported by the UK Biotechnology and Biological Sciences Research Council.

\section{References}

Al-Habori M (1994) Cell volume and ion transport regulation International Journal of Biochemistry 26 319-334

Arrazola A, Rota R, Hannaert P, Soler A and Garay RP (1993) Cell volume regulation in rat thymocytes Journal of Physiology 465 403-414

Bredderman PJ and Foote RH (1971a) Alterations of cell volume in bull spermatozoa by factors known to affect active cation transport Experimental Cell Research 66 190-196

Bredderman PJ and Foote RH (1971b) Factors stabilizing bull sperm cell volume and prolonging motility at high dilution Experimental Cell Research 66 458-464

Cheung RK, Grinstein S, Dosch H-D and Gelfand EW (1982) Volume regulation by human lymphocytes: characterization of the ionic basis for regulatory volume decrease Journal of Cell Physiology 112 189-196

Cooper TG (1986) The Epididymis, Sperm Maturation and Fertilisation. Springer-Verlag, Berlin

Curry MR (2000) Cryopreservation of semen from domestic livestock Reviews of Reproduction 5 46-52

Drevius L-O (1972) Bull spermatozoa as osmometers Journal of Reproduction and Fertility 28 29-39

Drevius L-O and Eriksson H (1966) Osmotic swelling of mammalian spermatozoa Experimental Cell Research 42 136-156

Foote RH (1970) Fertility of bull semen at high extension rates in Trisbuffered extenders Journal of Dairy Science 53 1475-1477

Gao DY, Ashworth E, Watson PF, Kleinhaus FW, Mazur P and Critser JK (1993) Hyperosmotic tolerance of human spermatozoa: separate effects of glycerol, sodium chloride, and sucrose on spermolysis Biology of Reproduction 49 112-123

Garay RP, Nazaret C, Hannaert PA and Cragoe EJ (1988) Demonstration of a $\left[\mathrm{K}^{+}, \mathrm{Cl}^{-}\right]$-cotransport system in human red cells by its sensitivity to [(dihydroindenyl)oxy]alkanoic acids: regulation of cell swelling and distinction from the bumetanide-sensitive $\left[\mathrm{Na}^{+}, \mathrm{K}^{+}, \mathrm{Cl}^{-}\right]$-cotransport system Molecular Pharmacology 33 696-701

Gilmore JA, Du J, Tao J, Peter AT and Critser JK (1996) Osmotic properties of boar spermatozoa and their relevance to cryopreservation Journal of Reproduction and Fertility 107 87-95

Gilmore JA, Liu J, Peter AT and Critser JK (1998) Determination of plasma membrane characteristics of boar spermatozoa and their relevance to cryopreservation Biology of Reproduction 58 28-36

Grinstein S, Rothstein A, Sarkadi B and Gelfand EW (1984) Responses of lymphocytes to anisotonic media: volume-regulating behavior American Journal of Physiology 246 C204-C215

Harrison RAP and Vickers SE (1990) Use of fluorescent probes to access membrane integrity in mammalian spermatozoa Journal of Reproduction and Fertility 88 343-352

Harrison RAP, Dott HM and Foster GC (1978) Effect of ionic strength, serum albumin and other macromolecules on the maintenance of motility and the surface of mammalian spermatozoa in a simple medium Journal of Reproduction and Fertility 52 65-73

Harrison RAP, Dott HM and Foster GC (1982) Bovine serum albumin, sperm motility, and the 'dilution effect' Journal of Experimental Zoology 222 81-88

Harrison RAP, Mairet B and Miller NGA (1993) Flow cytometric studies of bicarbonate-mediated $\mathrm{Ca}^{2+}$ influx in boar sperm populations Molecular Reproduction and Development 35 197-208

Hoffmann EK and Dunham PB (1995) Membrane mechanisms and intracellular signalling in cell volume recognition International Reviews of Cytology 161 173-262

Holt WV and North RD (1994) Effects of temperature and restoration of osmotic equilibrium during thawing on the induction of plasma membrane damaged in cryopreserved ram spermatozoa Biology of Reproduction $\mathbf{5 1}$ 414-424

Jentsch TJ and Günther W (1997) Chloride channels: an emerging molecular picture BioEssays 19 117-126

Johnson LA, Aalbers JG and Grooten HJG (1988) Artificial insemination of swine: fecundity of boar semen stored in Beltsville TS (BTS), Modified Modena (MM), or MR-A and inseminated on one, three and four days after collection Zuchthygiene 23 49-55

Kulkarni SB, Sauna ZE, Somlata V and Sitaramam V (1997) Volume regulation of spermatozoa by quinine-sensitive channels Molecular Reproduction and Development 46 535-550

Lang F, Busch GL, Ritter M, Völkl H, Waldegger S, Gulbins E and Häussinger D (1998) Functional significance of cell volume regulatory mechanisms Physiological Reviews 78 247-306

Meizel S (1997) Amino acid neurotransmitter receptor/chloride channels of mammalian sperm and the acrosome reaction Biology of Reproduction $\mathbf{5 6} 569-574$

O'Neill WC (1999) Physiological significance of volume-regulatory transporters American Journal of Physiology 276 C995-C1011

Parker JC (1993) In defense of cell volume? American Journal of Physiology 265 C1191-C1200

Petrunkina AM and Töpfer-Petersen E (2000) Heterogeneous osmotic behaviour in boar sperm populations and its relevance for detection of changes in plasma membrane Reproduction, Fertility and Development 12 297-305

Petrounkina AM, Harrison RAP, Petzoldt R, Weitze KF and TöpferPetersen E (2000) Cyclical changes in sperm volume during in vitro incubation under capacitating conditions: a novel boar sperm characteristic Journal of Reproduction and Fertility 118 283-293

Rodriguez-Gil JE and Rigau T (1996) Effects of ouabain on the response to osmotic changes in dog and boar spermatozoa Theriogenology 45 873-888

Sarkadi B and Parker JC (1991) Activation of ion transport pathways by changes in cell volume Biochimica et Biophysica Acta 1071 407-427

Sarkadi B, Cheung R, Mack E, Grinstein S, Gelfand EW and Rothstein A (1985) Cation and anion transport pathways in volume regulatory response of human lymphocytes to hyposmotic media American Journal of Physiology 248 C480-C487

Strange K, Emma F and Jackson PS (1996) Cellular and molecular physiology of volume-sensitive anion channels American Journal of Physiology 270 C711-C730

Yeung C-H, Sonnenberg-Riethmacher E and Cooper TG (1999) Infertile spermatozoa of c-ros tyrosine kinase receptor knockout mice show flagellar angulation and maturational defects in cell volume regulatory mechanisms Biology of Reproduction 61 1062-1069

Yeung C-H, Wagenfeld A, Nieschlag E and Cooper TG (2000) The cause of infertility of male c-ros tyrosine kinase receptor knockout mice Biology of Reproduction 63 612-618

Received 8 November 2000.

First decision 13 December 2000.

Accepted 17 April 2001. 